\title{
Comparison of pedicle screw-based dynamic stabilization and fusion surgery in the treatment of radiographic adjacent-segment degeneration: a retrospective analysis of single L5-S1 degenerative spondylosis covering 4 years
}

\author{
Yu Han, MM, Jianguang Sun, MM, Chenghan Luo, MM, Shilei Huang, MM, Liren Li, MM, \\ Xiang Ji, MM, Xiaozong Duan, MM, Zhenqing Wang, MM, and Guofu Pi, MM \\ Department of Orthopedics, the First Affiliated Hospital of Zhengzhou University, Zhengzhou, Henan Province, People's Republic \\ of China
}

OBJECTIVE Pedicle screw-based dynamic spinal stabilization systems (PDSs) were devised to decrease, theoretically, the risk of long-term complications such as adjacent-segment degeneration (ASD) after lumbar fusion surgery. However, to date, there have been few studies that fully proved that a PDS can reduce the risk of ASD. The purpose of this study was to examine whether a PDS can influence the incidence of ASD and to discuss the surgical coping strategy for L5S1 segmental spondylosis with preexisting L4-5 degeneration with no related symptoms or signs.

METHODS This study retrospectively compared 62 cases of L5-S1 segmental spondylosis in patients who underwent posterior lumbar interbody fusion $(n=31)$ or K-Rod dynamic stabilization $(n=31)$ with a minimum of 4 years' follow-up. The authors measured the intervertebral heights and spinopelvic parameters on standing lateral radiographs and evaluated preexisting ASD on preoperative MR images using the modified Pfirrmann grading system. Radiographic ASD was evaluated according to the results of radiography during follow-up.

RESULTS All 62 patients achieved remission of their neurological symptoms without surgical complications. The Kaplan-Meier curve and Cox proportional-hazards model showed no statistically significant differences between the 2 surgical groups in the incidence of radiographic ASD ( $p>0.05)$. In contrast, the incidence of radiographic ASD was 8.75 times $(95 \% \mathrm{Cl} 1.955-39.140 ; p=0.005)$ higher in the patients with a preoperative modified Pfirrmann grade higher than 3 than it was in patients with a modified Pfirrmann grade of 3 or lower. In addition, no statistical significance was found for other risk factors such as age, sex, and spinopelvic parameters.

CONCLUSIONS Pedicle screw-based dynamic spinal stabilization systems were not found to be superior to posterior lumbar interbody fusion in preventing radiographic ASD (L4-5) during the midterm follow-up. Preexisting ASD with a modified Pfirrmann grade higher than 3 was a risk factor for radiographic ASD. In the treatment of degenerative diseases of the lumbosacral spine, the authors found that both of these methods are feasible. Also, the authors believe that no extra treatment, other than observation, is needed for preexisting degeneration in L4-5 without any clinical symptoms or signs.

http://thejns.org/doi/abs/10.3171/2016.4.SPINE1679

KEY WORDS spinal fusion; pedicle screw-based dynamic spinal stabilization system; preexisting adjacent-segment degeneration; risk factor; adjacent-segment degeneration; lumbar; degenerative

$\mathrm{L}$ UMBAR spinal fusion surgery has grown in popularity dramatically over the past decade, and because of its high success rate it has served as the standard by which various disorders of the lumbar spine can be judged. However, fusion surgery alters endplate loading and increases adjacent-segment intradiscal pressures and range of motion. ${ }^{4}$ Thus, fusion surgery is considered a major risk factor for adjacent-segment degeneration (ASD).

ASD, an adverse sequela of lumbar arthrodesis, has been reported in many studies. ASD can be classified as symptomatic or radiographic. ${ }^{8}$ Symptomatic ASD is defined as new symptoms or signs that correspond to the

ABBREVIATIONS ASD = adjacent-segment degeneration; BMI = body mass index; LL = lumbar lordosis; ODI = Oswestry Disability Index; PDS = pedicle screw-based dynamic spinal stabilization system; PI = pelvic incidence; PLIF = posterior lumbar interbody fusion; $\mathrm{PT}=$ pelvic tilt; $\mathrm{SS}=$ sacral slope.

SUBMITTED January 14, 2016. ACCEPTED April 5, 2016.

INCLUDE WHEN CITING Published online June 24, 2016; DOI: 10.3171/2016.4.SPINE1679. 
adjacent segment, whereas radiographic ASD refers to radiographic changes that occur at the adjacent segment. ${ }^{29}$

Many studies have reported that fixated segments can predispose a patient to ASD. ${ }^{21,23}$ In an attempt to address ASD associated with lumbar fusion surgery, dynamic surgical devices have been developed. Two widely used dynamic modalities are 1) a pedicle screw-based dynamic spinal stabilization system (PDS) and 2) total disc arthroplasty. The developers of these approaches believed that adjacent-segment biomechanics would be affected less if the motion of the instrumented level was partially preserved.

PDSs such as the Dynesys dynamic stabilization system, the Isobar, and the K-Rod have been widely used in the treatment of lumbar diseases. Despite clinical outcomes being similar for both PDSs and fusion surgery, there is no concrete clinical evidence to support the hypothesis that a PDS can reduce the risk of ASD. In addition, the indications for PDS and its surgical strategies remain controversial.

The purposes of this study were to examine whether a PDS can influence the incidence of ASD and to discuss the surgical strategy for L5-S1 segmental spondylosis with L4-5 preexisting degeneration with no symptoms or signs.

\section{Methods}

\section{Study Design and Patient Population}

This was a retrospective cohort study. We compared outcomes of using the K-Rod dynamic stabilization system (Paonan Biotech, Inc.) with those of posterior lumbar interbody fusion (PLIF) in the treatment of lumbar spondylosis in patients for whom follow-up was a minimum of 4 years. The medical files and radiographs of 79 patients with L5-S1 segmental spondylosis who underwent lumbar surgery at the First Affiliated Hospital of Zhengzhou University between January 2011 and January 2012 were analyzed. All data were reviewed after obtaining approval from our institutional review board.

Inclusion criteria for both groups included lumbar disc herniation with spinal canal stenosis, age of $\geq 20$ years at the time of surgery, no proof of instability in L5-S1 or adjacent levels, and no history of lumbar surgery. None of the patients included in the study had a diagnosis of spinal neoplasm, fracture, infection, or pars defects. Also, osteoporosis was an exclusion criterion. Nine patients who received discectomy without internal fixation or had a major lumbar deformity, such as scoliosis with a Cobb angle $\geq 15^{\circ}$ or kyphosis with a Cobb angle $\geq 10^{\circ}$, were excluded, and 8 individuals were excluded because their follow-up period was less than 4 years.

\section{Surgical Technique}

All procedures were performed at the First Affiliated Hospital of Zhengzhou University by the senior author (G.P.). After the induction of anesthesia, each patient was placed in the prone position, and perioperative antibiotics were administrated before skin incision. The posterior midline approach was used in both groups. Decompression was conducted based on patient symptoms and preoperative MRI and CT scan results. For patients in the
PLIF group, a polyetheretherketone (PEEK) cage (Stryker Spine) packed with posterior bone graft (laminar bone chips) was inserted in the disc space after disc removal and preparation of the endplates, whereas discectomy alone was conducted for patients in the PDS group. The patients who underwent PLIF were treated by using titanium alloy pedicle screws and rods (Stryker Spine). Titanium alloy pedicle screws and PEEK-coated cable rods (Paonan Biotech, Inc.) were implanted in the patients in the PDS group.

\section{Clinical and Radiographic Evaluations}

Clinical outcomes were assessed by using the Oswestry Disability Index (ODI) before surgery and postoperatively at 3 months, 6 months, and annually up to 4 years after surgery.

Standing lumbar anteroposterior and lateral radiographs were acquired before surgery and postoperatively at 3 months, 6 months, and annually up to 4 years after surgery. Two independent radiologists evaluated the radiographs. Lumbar lordosis (LL), pelvic incidence (PI), and sacral slope (SS) were measured on lumbar lateral radiographs in an attempt to investigate the correlation between spinopelvic alignment and ASD. The intervertebral space height was defined as the average value of the anterior, central, and posterior disc heights and was measured at the adjacent segment (L4-5). Two independent reviewers were asked to define preexisting ASD on preoperative MR images according to the modified Pfirrmann grading system. ${ }^{7}$

\section{Criteria for Radiographic ASD}

Radiographic ASD was defined as follows: 1) disc height reduction of more than $3 \mathrm{~mm}$ seen on a lateral radiograph, 2) L-4 vertebral slippage of more than $3 \mathrm{~mm}$ compared with the preoperative condition seen on lateral radiographs, 3) and progression of osteophytes and endplate sclerosis compared with the preoperative condition.

\section{Statistical Analysis}

The incidence of radiographic ASD was analyzed by using life-table methods and construction of Kaplan-Meier survivorship curves.

Risk factors for developing radiographic ASD were evaluated by using the log-rank test, and the Cox proportional-hazards model was used to adjust for confounding variables. The following variables were included in this analysis: age, sex, preoperative modified Pfirrmann grade, the type of implants, LL, pelvic tilt (PT), and PI. The t-test and ANOVA were used to analyze baseline data, changes in relative disc height, ODIs, and spinopelvic parameters.

Statistical analyses were performed with the use of SPSS 21.0 (SPSS, Inc.) and GraphPad Prism 5 (GraphPad Software, Inc.). $\mathrm{p}$ values $<0.05$ based on a 2 -sided hypothesis test were considered significant.

\section{Result \\ Demographic Results}

In our database, a total of 62 patients (30 men, 32 wom- 
en) with a mean age of 43.2 years (range 21-63 years) met the inclusion criteria. PLIF was performed in 31 patients (12 men, 19 women). The mean age of patients in the PLIF group at the time of surgery was 46.9 years (range 24-63 years). The PDS group consisted of 31 subjects (18 male, 13 female) with a mean age of 39.4 years (range 21-63 years).

There were no statistically significant differences in baseline demographics between the PDS and PLIF groups in terms of sex, ethnicity, mean body mass index (BMI), preoperative modified Pfirrmann grade, LL, PI, or SS (Table 1). No surgical complications, including screw loosening or dislodging, were reported in the 2 groups, and no revision surgery was needed.

Twenty patients (32.26\%) presented with radiographic ASD during the follow-up period. No symptomatic ASD was noted during the follow-up period. There were no statistically significant differences in the clinical results between the treatments (Table 1).

\section{Risk-Factor Analysis}

We used Kaplan-Meier curves to analyze all available data and to predict the risk factors for ASD. In terms of preventing radiographic ASD, PDS showed no superiority over PLIF in univariate analysis using the log-rank test ( $\mathrm{p}$ $=0.6696)$. The hazard ratio of the 2 groups was 0.8253 (95\% CI 0.3416-1.994) (Fig. 1).

Patients with preexisting ASD (modified Pfirrmann grade $\leq 3$ ) experienced a significantly lower incidence of

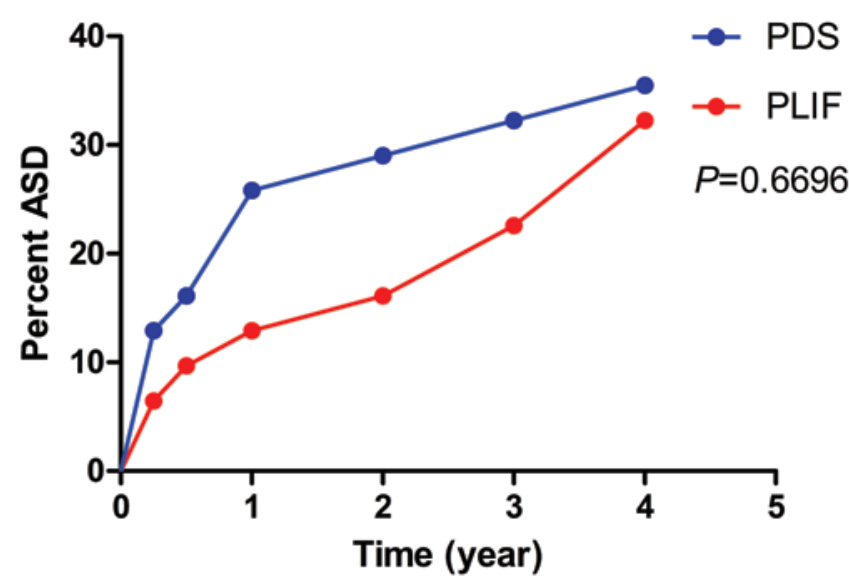

FIG. 1. Kaplan-Meier curve of radiographic ASD in the 2 surgical groups. Figure is available in color online only.

radiographic ASD than those with preexisting ASD (modified Pfirrmann grade $>3)(\mathrm{p}=0.0005)$. The hazard ratio was 4.958 (95\% CI 2.003-12.27) (Fig. 2).

In addition, no statistically significant differences were detected for risk factors such as age, sex, LL, PI, or SS.

The results of univariate analysis were corroborated by the Cox proportional-hazards model (Table 2). In the risk-factor analysis, compared with the patients with a modified Pfirrmann grade of $\leq 3$ for an adjacent segment, the incidence of radiographic ASD was 8.75 times $(95 \%$

TABLE 1. Baseline demographic and preoperative radiographic data*

\begin{tabular}{|c|c|c|c|}
\hline Demographic or Preop Data & PDS Group $(n=31)$ & PLIF Group $(n=31)$ & p Value \\
\hline Mean age (yrs) & $39.41 \pm 9.56$ & $46.90 \pm 9.21$ & $0.003 \dagger$ \\
\hline $\operatorname{Sex}(M / F)$ & $18: 13$ & $12: 19$ & $0.127 \ddagger$ \\
\hline Mean BMI $\left(\mathrm{kg} / \mathrm{m}^{2}\right)$ & $23.13 \pm 2.34$ & $23.71 \pm 2.55$ & 0.356 \\
\hline L4-5 preop modified Pfirrmann grade (no.) & & & $0.580 \ddagger$ \\
\hline 1 & 1 & 0 & \\
\hline 2 & 7 & 3 & \\
\hline 3 & 7 & 8 & \\
\hline 4 & 7 & 11 & \\
\hline 5 & 4 & 3 & \\
\hline 6 & 5 & 6 & \\
\hline 7 & 0 & 0 & \\
\hline 8 & 0 & 0 & \\
\hline Preop L-4 vertebral slip (no.) & 0 & 0 & - \\
\hline \multicolumn{4}{|l|}{ Mean ODI } \\
\hline Preop & $43.94 \pm 11.42$ & $46.52 \pm 14.10$ & 0.432 \\
\hline Last follow-up & $8.26 \pm 2.67$ & $8.19 \pm 2.65$ & 0.924 \\
\hline Mean LL $\left(^{\circ}\right)$ & $37.56 \pm 9.81$ & $40.38 \pm 13.01$ & 0.340 \\
\hline Mean PT $\left({ }^{\circ}\right)$ & $12.02 \pm 7.87$ & $13.25 \pm 8.23$ & 0.552 \\
\hline Mean $\mathrm{PI}\left({ }^{\circ}\right)$ & $41.21 \pm 8.96$ & $42.86 \pm 8.29$ & 0.454 \\
\hline Mean SS $\left(^{\circ}\right)$ & $29.50 \pm 8.74$ & $29.63 \pm 6.47$ & 0.947 \\
\hline
\end{tabular}




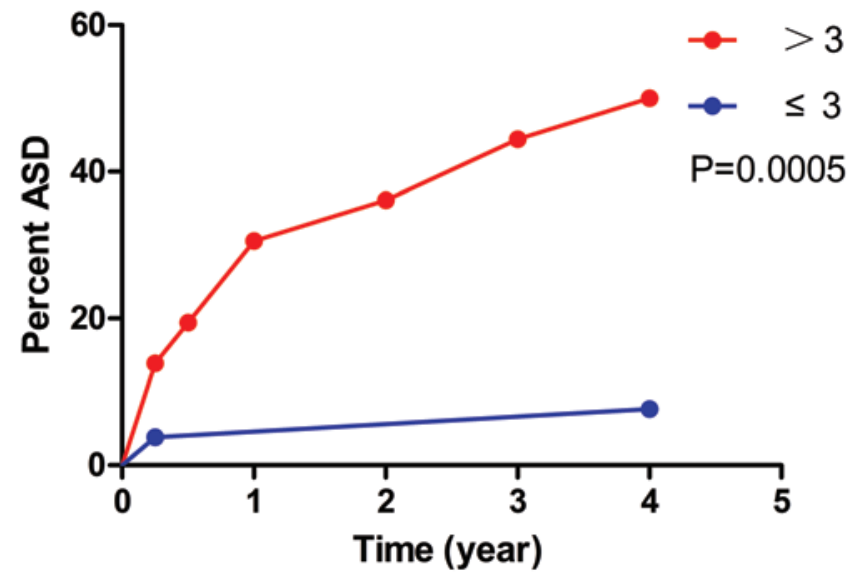

FIG. 2. Kaplan-Meier curve of radiographic ASD of different preexisting modified Pfirmann degeneration grades. Figure is available in color online only.

CI 1.955-39.140; $\mathrm{p}=0.005$ ) higher in the patients with a modified Pfirrmann grade $>3$. Variables such as surgical treatment, age, sex, LL, PI, and SS did not present statistical significance in the Cox proportional-hazards model.

\section{Radiograph Analysis}

According to radiographic analyses of the 31 patients in the PDS group, a reduction in disc height of more than $3 \mathrm{~mm}$ was documented in $9(29.03 \%)$ cases, and an L-4 vertebral slip greater than $3 \mathrm{~mm}$ occurred in 2 patients (6.45\%). Among the patients who underwent PLIF, 7 (22.58\%) experienced a decrease in intervertebral height of more than $3 \mathrm{~mm}$, and an L-4 vertebral slip was observed in 5 patients $(16.12 \%)$. No significant progress of osteophytes or endplate sclerosis was found in either of the 2 groups.

Over the follow-up period, the height of the adjacent disc (L4-5) in both groups was gradually reduced ( $\mathrm{p}=$ 0.000 ). The downward trend of relative disc height was detected in both surgical groups (Pillai's trace $=0.013 ; \mathrm{F}=$

TABLE 2. Cox proportional hazards for risk of ASD

\begin{tabular}{|c|c|c|c|c|}
\hline Factor & $\begin{array}{c}\text { No. of } \\
\text { Patients }\end{array}$ & $\begin{array}{l}\text { Hazard } \\
\text { Ratio }\end{array}$ & $95 \% \mathrm{Cl}$ & p Value \\
\hline Age & & & & 0.277 \\
\hline$\leq 42 \mathrm{yrs}$ & 32 & & & \\
\hline$>42$ yrs & 30 & 1.877 & $0.604-5.839$ & \\
\hline Treatment & & & & 0.291 \\
\hline PDS & 31 & & & \\
\hline PLIF & 31 & 0.556 & $0.187-1.651$ & \\
\hline $\begin{array}{l}\text { L4-5 preop modified } \\
\text { Pfirrmann grade }\end{array}$ & & & & 0.005 \\
\hline$\leq 3$ & 26 & & & \\
\hline$>3$ & 36 & 8.747 & $1.955-39.140$ & \\
\hline Sex & & & & 0.459 \\
\hline Male & 30 & & & \\
\hline Female & 32 & 1.412 & $0.566-3.519$ & \\
\hline
\end{tabular}

$0.125, \mathrm{p}=0.993)$. Furthermore, there were no statistically significant differences between the PDS and PLIF groups in the changes in relative disc height $(\mathrm{p}=0.347)$ (Fig. 3).

In 2-way repeated-measures ANOVA, we found no statistically significant differences between the groups in preoperative and postoperative LL, PT, PI, or SS.

\section{Illustrative Cases}

A 33-year-old woman with L5-S1 disc herniation and spinal canal stenosis underwent K-Rod fixation (Fig. 4A-C). Her initial symptoms were low-back and left lower limb pain. Her preoperative degeneration at L4-5 was at modified Pfirrmann Grade 6. Her recovery progressed smoothly after surgery, but a reduction in L4 -5 intervertebral height $(>3 \mathrm{~mm})$ was observed 6 months after surgery. In another case-a 56-year-old woman with severe low-back and bilateral lower extremity pain for nearly 4 months who was diagnosed with lumbar spondylosisthe patient underwent PLIF (Fig. 4D-F). Her symptoms resolved after surgical intervention. There was no radiographic indication of ASD during her 4-year follow-up period.

\section{Discussion}

Because the diagnosis of radiographic ASD remains controversial, the incidence of radiographic ASD after lumbar fusion surgery has varied widely among published reports, ranging from $4.8 \%$ to $92.2 \% .{ }^{29}$ Studies of symptomatic ASD have reported incidences that range from $0 \%$ to $30.3 \% .^{29}$ In this study, 20 (32.25\%) of 62 patients in the PLIF and PDS groups had postoperative radiographic degenerative changes ( 9 [29.03\%] of 31 in the PDS group; 11 [35.48\%] of 31 in the PLIF group). Previous studies have documented a number of risk factors for ASD, such as age, multilevel fusion, BMI at admission, spinal sagittal alignment, and menopause. ${ }^{14,21,23,24}$

A significant risk factor in determining ASD is preexisting degeneration at an adjacent level, including spinal

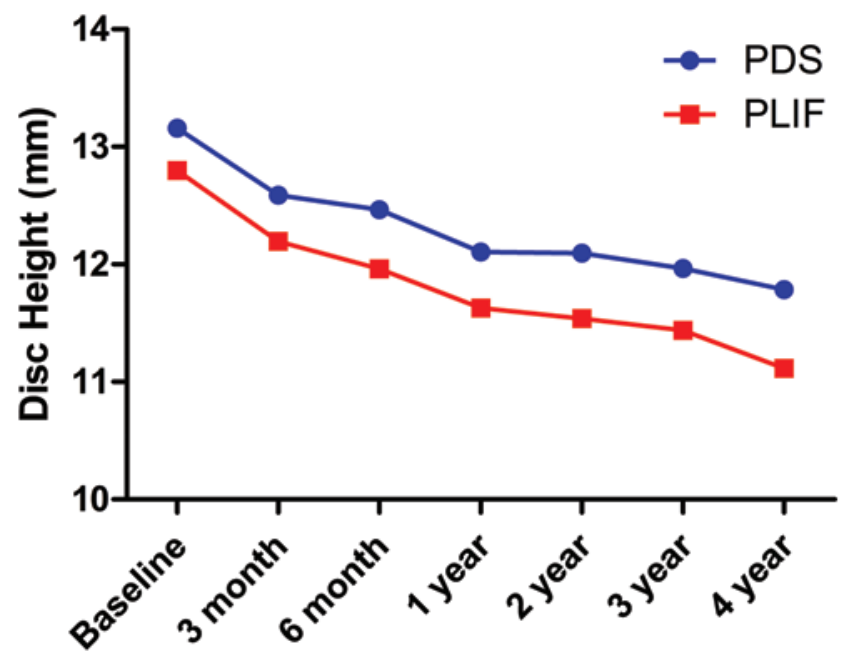

FIG. 3. Changes in relative $L 4-5$ disc height. Figure is available in color online only. 

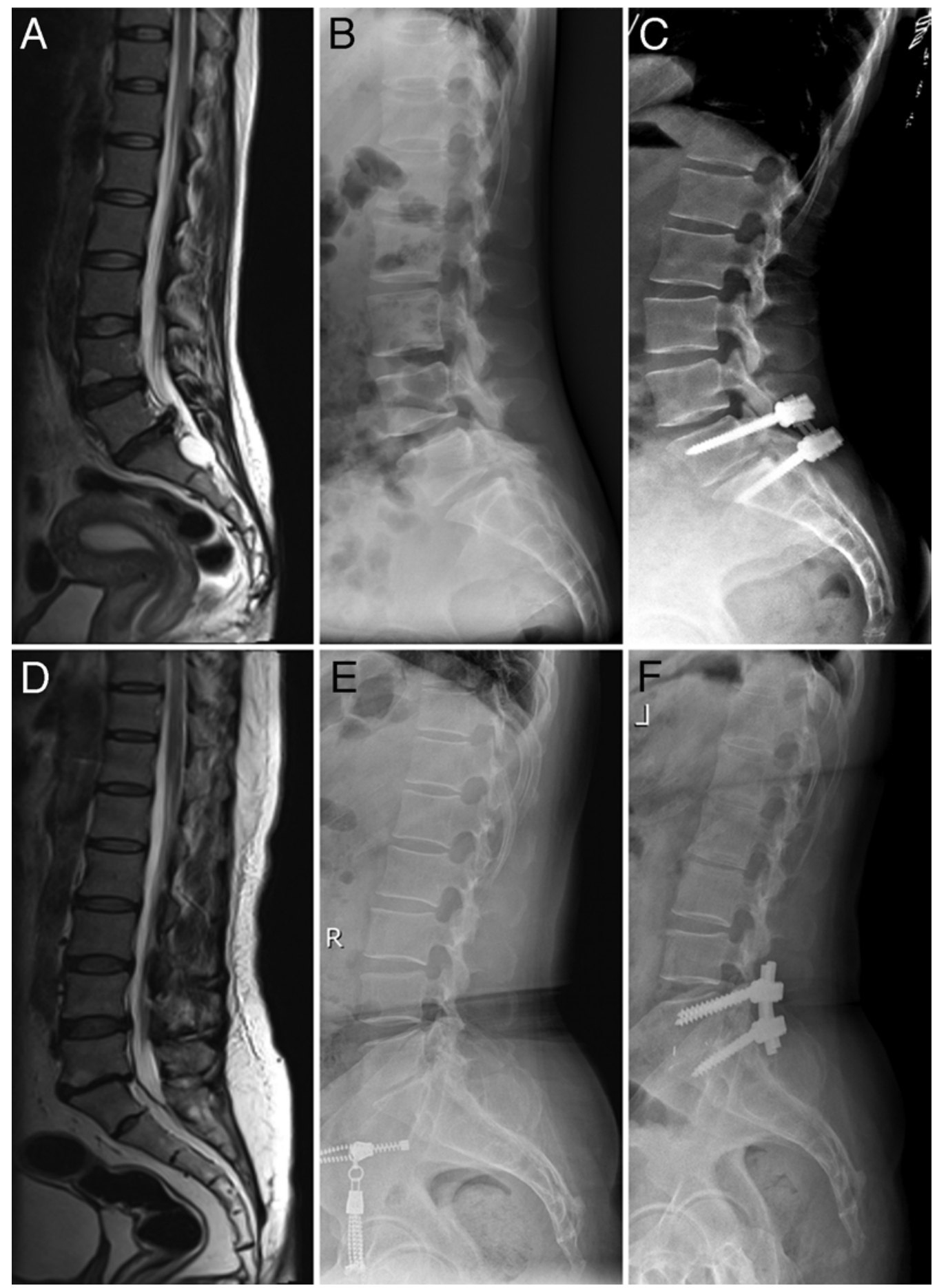

FIG. 4. A: Preoperative sagittal MR image. B: Preoperative lateral radiograph. C: Lateral radiograph 6 months after surgery showing reduction of the L4-5 intervertebral height of more than $3 \mathrm{~mm}$. D: Preoperative sagittal MR image. E: Preoperative lateral radiograph. F: Lateral radiograph 4 years after surgery showing no indication of radiographic ASD.

stenosis and facet and disc degeneration. ${ }^{15}$ Based on the hypothesis that degenerative aging factors have a greater influence than biomechanical factors, $\mathrm{Li}$ et al. ${ }^{17}$ claimed that adjacent segments with preexisting degeneration have a higher chance of ASD than do normal discs. According to MRI results, preexisting facet degeneration might be associated with a higher risk of ASD. ${ }^{13}$ Furthermore, some authors have suggested that surgery should be carefully considered in patients whose preoperative modified Pfirrmann grade is higher than $3 .{ }^{18}$ However, Choi et al. ${ }^{2}$ found that preexisting L5-S1 degeneration did not affect clinical or radiographic results after L4-5 fusion. In line with re- 
sults of the studies by Li et al. ${ }^{17}$ and Liang et al.. ${ }^{18}$ we found that patients who had adjacent segments with preexisting degeneration (at a modified Pfirrmann grade $>3$ ) were more likely to experience accelerated progression of ASD.

However, many reports have regarded the fusion technique as the most important risk factor for ASD. ${ }^{1,13,14,21,23,26}$ A systematic review conducted by Lawrence et al. ${ }^{11}$ revealed that development of symptomatic ASD after lumbar fusion occurs at a mean annual incidence of $0.6 \%-$ $3.9 \%$. However, animal and cadaver studies have found increased range of motion and intervertebral stress in the adjacent segments after spinal arthrodesis. ${ }^{3,12}$ The notion that spinal fusion changes the biomechanics of adjacent levels has played a large role in the development of motionpreserving techniques in lumbar surgery. Clinical studies have shown that PDS offers satisfactory short-term clinical outcomes and suggested that PDS is a possible alternative to PLIF, ${ }^{9,19}$ but little research has found that it helps to prevent ASD. In a 4-year follow-up study, Schaeren et al. ${ }^{27}$ found that degeneration in adjacent motion segments progresses after Dynesys dynamic stabilization. In our study, no significant differences in the incidence of radiographic ASD were detected between the 2 groups.

In addition, spinopelvic alignment has been regarded as one of the major risk factors in recent studies. In a retrospective case-control study of 101 patients who underwent PLIF and more than 10 years' follow-up, Nakashima et al. ${ }^{23}$ found that a higher degree of PI is a risk factor for developing early-onset radiographic ASD and that creating the appropriate LL in PLIF is important for preventing ASD. The results of a modeling study performed by Senteler et al. ${ }^{28}$ suggested that the correlation between $\triangle \mathrm{PI}-\mathrm{LL}$ (the difference between PI and LL) and ASD can be ascribed to the higher shear stresses before and after fusion. In the transition from upright to $30^{\circ}$ flexed posture, they observed that average shear-force changes were $32 \%$ higher at L3-4 and 14\% higher at L4-5 in the alignments that were susceptible to ASD..$^{28}$ In addition, a PT of $>21^{\circ}$ and an SS of $<39^{\circ}$ were perceived as factors for elevated risk for symptomatic ASD. ${ }^{5}$ However, with cases of short fixation, Masevnin et al. ${ }^{21}$ failed to find statistically significant dependency between ASD and sagittal imbalance. In our study, there were no significant differences in spinal sagittal alignment between patients with and those without ASD in both groups. Because the patients involved in our research all underwent single-level fixation, the untreated segments could compensate for the sagittal balance disturbance.

According to the results of our study, adjacent disc degeneration that already existed before the surgical treatment played an important role in the progression of ASD. Also, preexisting ASD always made an appropriate surgical indication difficult to achieve. Additional decompression at adjacent segments was regarded as an option by some surgeons for tackling this problem. By studying postoperative radiographs, Gard et al. ${ }^{6}$ concluded that laminectomy above the index level did not increase ASD beyond its natural incidence. However, more researchers held the opposite view that simultaneous decompression without fusion at the adjacent segments was futile or, even worse, that there was a possibility that it would induce
ASD. ${ }^{8,22}$ Clinging to the belief that disrupting the integrity of posterior spinal elements can jeopardize lumbar spine stability, we eliminated this option in all cases.

The hybrid use of PDS and fusion, designed to preserve motion of the spine and share the loads adjacent to fixed segments in the setting of fusion, recently became a focus of clinical studies..$^{10,16,20,25}$ Despite the reported satisfactory clinical outcomes, we did not adopt the hybrid method because 1) hybrid instruments are used to substitute for fusion when treating symptomatic ASD, and 2) hybrid instruments are too costly.

There was no strong evidence of a correlation between radiographic ASD and symptomatic ASD. We did not perform aggressive treatment to the preexisting ASD at L4-5 because of the shorter fixation and fusion principle. Only if radiographic ASD is accompanied by related symptoms or signs will further treatment be taken into consideration.

Limitations of our study include the retrospective nature of the analysis, the small numbers of cases enrolled, and the relatively short follow-up period. In addition, postoperative MR images, which might have affected our evaluation of ASD, were not available for all of the patients. Last, we also acknowledge the limitations introduced by clinical heterogeneity. Therefore, further long-term studies are needed to find the precise risk factors for ASD and evaluate the efficiency of PDS.

\section{Conclusions}

PDS showed no superiority over PLIF in preventing radiographic ASD in middle-term follow-up. Preexisting ASD at a modified Pfirrmann grade greater than 3 was a risk factor for radiographic ASD. For the treatment of degenerative diseases in the lumbosacral spine, we found that both PDS and PLIF are feasible. Because the correlation between radiographic degeneration and clinical symptoms is not clear, we suggest that no medical treatment, other than observation, is needed for preexisting L4-5 degeneration without any related symptoms or signs.

\section{References}

1. Chen BL, Wei FX, Ueyama K, Xie DH, Sannohe A, Liu SY: Adjacent segment degeneration after single-segment PLIF: the risk factor for degeneration and its impact on clinical outcomes. Eur Spine J 20:1946-1950, 2011

2. Choi KC, Shim HK, Kim JS, Lee SH: Does pre-existing L5S1 degeneration affect outcomes after isolated L4-5 fusion for spondylolisthesis? J Orthop Surg 10:39, 2015

3. Cole TC, Burkhardt D, Ghosh P, Ryan M, Taylor T: Effects of spinal fusion on the proteoglycans of the canine intervertebral disc. J Orthop Res 3:277-291, 1985

4. Cunningham BW, Kotani Y, McNulty PS, Cappuccino A, McAfee PC: The effect of spinal destabilization and instrumentation on lumbar intradiscal pressure: an in vitro biomechanical analysis. Spine (Phila Pa 1976) 22:2655-2663, 1997

5. Di Martino A, Quattrocchi CC, Scarciolla L, Papapietro N, Beomonte Zobel B, Denaro V: Estimating the risk for symptomatic adjacent segment degeneration after lumbar fusion: analysis from a cohort of patients undergoing revision surgery. Eur Spine J 23 (Suppl 6):693-698, 2014

6. Gard AP, Klopper HB, Doran SE, Hellbusch LC: Analysis of adjacent segment degeneration with laminectomy above a fused lumbar segment. J Clin Neurosci 20:1554-1557, 2013 
7. Griffith JF, Wang YX, Antonio GE, Choi KC, Yu A, Ahuja AT, et al: Modified Pfirrmann grading system for lumbar intervertebral disc degeneration. Spine (Phila Pa 1976) 32:E708-E712, 2007

8. Hikata T, Kamata M, Furukawa M: Risk factors for adjacent segment disease after posterior lumbar interbody fusion and efficacy of simultaneous decompression surgery for symptomatic adjacent segment disease. J Spinal Disord Tech 27:70-75, 2014

9. Hoppe S, Schwarzenbach O, Aghayev E, Bonel H, Berlemann U: Long-term outcome after monosegmental L4/5 stabilization for degenerative spondylolisthesis with the Dynesys device. J Spinal Disord Tech [epub ahead of print], 2012

10. Hudson WR, Gee JE, Billys JB, Castellvi AE: Hybrid dynamic stabilization with posterior spinal fusion in the lumbar spine. SAS J 5:36-43, 2011

11. Lawrence BD, Wang J, Arnold PM, Hermsmeyer J, Norvell DC, Brodke DS: Predicting the risk of adjacent segment pathology after lumbar fusion: a systematic review. Spine (Phila Pa 1976) 37 (22 Suppl):S123-S132, 2012

12. Lee CK: Accelerated degeneration of the segment adjacent to a lumbar fusion. Spine (Phila Pa 1976) 13:375-377, 1988

13. Lee CS, Hwang CJ, Lee SW, Ahn YJ, Kim YT, Lee DH, et al: Risk factors for adjacent segment disease after lumbar fusion. Eur Spine J 18:1637-1643, 2009

14. Lee JC, Kim Y, Soh JW, Shin BJ: Risk factors of adjacent segment disease requiring surgery after lumbar spinal fusion: comparison of posterior lumbar interbody fusion and posterolateral fusion. Spin (Phila Pa 1976) 39:E339-E345, 2014

15. Lee MJ, Dettori JR, Standaert CJ, Ely CG, Chapman JR: Indication for spinal fusion and the risk of adjacent segment pathology: does reason for fusion affect risk? A systematic review. Spine (Phila Pa 1976) 37 (22 Suppl):S40-S51, 2012

16. Lee SE, Jahng TA, Kim HJ: Hybrid surgery combined with dynamic stabilization system and fusion for the multilevel degenerative disease of the lumbosacral spine. Int J Spine Surg 9:45, 2015

17. Li C, He Q, Tang Y, Ruan D: The fate of adjacent segments with pre-existing degeneration after lumbar posterolateral fusion: the influence of degenerative grading. Eur Spine J 24:2468-2473, 2015

18. Liang J, Dong Y, Zhao H: Risk factors for predicting symptomatic adjacent segment degeneration requiring surgery in patients after posterior lumbar fusion. J Orthop Surg 9:97, 2014

19. Liu C, Wang L, Tian JW: Early clinical effects of the Dynesys system plus transfacet decompression through the Wiltse approach for the treatment of lumbar degenerative diseases. Med Sci Monit 20:853-859, 2014

20. Maserati MB, Tormenti MJ, Panczykowski DM, Bonfield CM, Gerszten PC: The use of a hybrid dynamic stabilization and fusion system in the lumbar spine: preliminary experience. Neurosurg Focus 28(6):E2, 2010

21. Masevnin S, Ptashnikov D, Michaylov D, Meng H, Smekalenkov O, Zaborovskii N: Risk factors for adjacent segment disease development after lumbar fusion. Asian Spine J 9:239-244, 2015
22. Miyagi M, Ikeda O, Ohtori S, Tsuneizumi Y, Someya Y, Shibayama M, et al: Additional decompression at adjacent segments leads to adjacent segment degeneration after PLIF. Eur Spine J 22:1877-1883, 2013

23. Nakashima H, Kawakami N, Tsuji T, Ohara T, Suzuki Y, Saito T, et al: Adjacent segment disease after posterior lumbar interbody fusion: based on cases with a minimum of 10 years of follow-up. Spine (Phila Pa 1976) 40:E831-E841, 2015

24. Ou CY, Lee TC, Lee TH, Huang YH: Impact of body mass index on adjacent segment disease after lumbar fusion for degenerative spine disease. Neurosurgery 76:396-402, 2015

25. Prud'homme M, Barrios C, Rouch P, Charles YP, Steib JP, Skalli W: Clinical outcomes and complications after pedicleanchored dynamic or hybrid lumbar spine stabilization: a systematic literature review. J Spinal Disord Tech 28:E439E448, 2015

26. Radcliff K, Curry P, Hilibrand A, Kepler C, Lurie J, Zhao W, et al: Risk for adjacent segment and same segment reoperation after surgery for lumbar stenosis: a subgroup analysis of the Spine Patient Outcomes Research Trial (SPORT). Spine (Phila Pa 1976) 38:531-539, 2013

27. Schaeren S, Broger I, Jeanneret B: Minimum four-year follow-up of spinal stenosis with degenerative spondylolisthesis treated with decompression and dynamic stabilization. Spine (Phila Pa 1976) 33:E636-E642, 2008

28. Senteler M, Weisse B, Snedeker JG, Rothenfluh DA: Pelvic incidence-lumbar lordosis mismatch results in increased segmental joint loads in the unfused and fused lumbar spine. Eur Spine J 23:1384-1393, 2014

29. Xia XP, Chen HL, Cheng HB: Prevalence of adjacent segment degeneration after spine surgery: a systematic review and meta-analysis. Spine (Phila Pa 1976) 38:597-608, 2013

\section{Disclosures}

The authors report no conflict of interest concerning the materials or methods used in this study or the findings specified in this paper.

\section{Author Contributions}

Conception and design: Pi, Han. Acquisition of data: Han, Li, Ji, Duan, Wang. Analysis and interpretation of data: Han. Drafting the article: Han. Critically revising the article: Han. Reviewed submitted version of manuscript: Han. Statistical analysis: Han. Administrative/technical/material support: Han, Sun, Luo, Huang. Study supervision: Pi, Han.

\section{Correspondence}

Guofu Pi, Department of Orthopedics, First Affiliated Hospital of Zhengzhou University, No. 1 Jianshe Dong Rd., Zhengzhou, Henan Province 450052, People's Republic of China. email: gfpspine@sina.com. 